\title{
Nodal Solution for a Kirchhoff-Type Problem in $\mathbb{R}^{N}$
}

\section{Leilei Sha}

College of Science, University of Shanghai for Science and Technology, Shanghai, China

Email: 845935050@qq.com

How to cite this paper: Sha, L.L. (2020) Nodal Solution for a Kirchhoff-Type Problem in $\mathbb{R}^{N}$. Applied Mathematics, 11, 42-52. https://doi.org/10.4236/am.2020.111005

Received: November 30, 2019

Accepted: January 7, 2020

Published: January 10, 2020

Copyright $\odot 2020$ by author(s) and Scientific Research Publishing Inc. This work is licensed under the Creative Commons Attribution International License (CC BY 4.0).

http://creativecommons.org/licenses/by/4.0/

\section{Abstract}

In this paper, we study the existence of nodal solutions of the following general Schödinger-Kirchhoff type problem:

$$
\begin{aligned}
& \left(a+b \int_{\mathbb{R}^{N}} g^{2}(u)|\nabla u|^{2} \mathrm{~d} x\right)\left[-\operatorname{div}\left(g^{2}(u) \nabla u\right)+g^{\prime}(u) g(u)|\nabla u|^{2}\right]+u \\
& =h(u), \text { in } \mathbb{R}^{N},
\end{aligned}
$$

where $a, b>0, \quad N \geq 3, g: \mathbb{R} \rightarrow \mathbb{R}^{+}$is an even differential function and $g^{\prime}(s) \geq 0$ for all $s \geq 0, h: \mathbb{R} \rightarrow \mathbb{R}$ is an odd differential function. These equations are related to the generalized quasilinear Schödinger equations:

$$
-\operatorname{div}\left(g^{2}(u) \nabla u\right)+g^{\prime}(u) g(u)|\nabla u|^{2}+V(x) u=h(u) \text {, in } \mathbb{R}^{N} .
$$

Because the general Schödinger-Kirchhoff type problem contains the nonlocal term, it implies that the equation $(K P 1)$ is no longer a pointwise identity and is very different from classical elliptic equations. By introducing a variable replacement, we first prove that $(K P 1)$ is equivalent to the following problem:

$$
-\left(a+b \int_{\mathbb{R}^{N}}|\nabla v|^{2} \mathrm{~d} x\right) \Delta v+\frac{G^{-1}(v)}{g\left(G^{-1}(v)\right)}=\frac{h\left(G^{-1}(v)\right)}{g\left(G^{-1}(v)\right)}, \text { in } \mathbb{R}^{N},
$$

where $G(s)=\int_{0}^{s} g(t) \mathrm{d} t$ and $G^{-1}$ is the inverse of $G$. Next, we prove that $(K P 2)$ is equivalent to the following system with respect to $(v, \mu) \in \mathbb{R}^{N} \times \mathbb{R}^{+}$:

$$
\left\{\begin{array}{l}
-\Delta v+\frac{G^{-1}(v)}{g\left(G^{-1}(v)\right)}=\frac{h\left(G^{-1}(v)\right)}{g\left(G^{-1}(v)\right)}, \\
\mu-a-b \mu^{\frac{N-2}{2}} \int_{\mathbb{R}^{N}}|\nabla v|^{2} \mathrm{~d} x=0 .
\end{array}\right.
$$

For every integer $k>0$, radial solutions of (KP1) with exactly $k$ nodes are obtained by dealing with the system $(S)$ under some appropriate assumptions. Moreover, this paper established the nonexistence results if $N \geq 4$ and $b$ is sufficiently large. 


\section{Keywords}

Sign-Changing Solutions, Nonlocal Operator, Kirchhoff-Type Equations

\section{Introduction}

In this paper, we consider the existence of nodal solutions for the following generalized quasilinear Schödinger-Kirchhoff type problem:

$$
\begin{aligned}
& \left(a+b \int_{\mathbb{R}^{N}} g^{2}(u)|\nabla u|^{2} \mathrm{~d} x\right)\left[-\operatorname{div}\left(g^{2}(u) \nabla u\right)+g^{\prime}(u) g(u)|\nabla u|^{2}\right]+V(x) u \\
& =h(u), \text { in } \mathbb{R}^{N}
\end{aligned}
$$

where $a, b>0, N \geq 3 . g: \mathbb{R} \rightarrow \mathbb{R}^{+}$is an even differential function and $g^{\prime}(s) \geq 0$ for all $s \geq 0, h: \mathbb{R} \rightarrow \mathbb{R}$ is an odd differential function, the potential $V(x): \mathbb{R}^{N} \rightarrow \mathbb{R}$ is positive in $\mathbb{R}^{N}$. It is necessary pointing out that we only consider the potential $V(x)=1$.

These equations are related to the quasilinear Schödinger (QS) equations

$$
i \partial_{t} z=-\Delta z+W(x) z-h(z)-\kappa \Delta l\left(|z|^{2}\right) l^{\prime}\left(|z|^{2}\right) z,
$$

where $z: \mathbb{R} \times \mathbb{R}^{N} \rightarrow \mathbb{C}, W: \mathbb{R}^{N} \rightarrow \mathbb{R}$ is a given potential, $\kappa$ is a real constant and $h, l: \mathbb{R} \rightarrow \mathbb{R}$ are suitable functions. Set $z(t, x)=\exp (-i E t) u(x)$, where $E \in \mathbb{R}$ and $u$ is a real function, (QS) can be reduce to the corresponding equation of elliptic type (see [1]):

$$
-\Delta u+V(x) u-\kappa \Delta l\left(|u|^{2}\right) l^{\prime}\left(|u|^{2}\right) u=h(u), x \in \mathbb{R}^{N} .
$$

The form of the above equation has derived as models of several physical phenomena corresponding to various types of $l(s)$. For instance, the case $l(s)=s$ models the time evolution of the condensate wave function in super-fluid film and are called the superfluid film equation in the fluid mechanics by Kurihara [2]. In the case $l(s)=(1+s)^{\frac{1}{2}}$ models the self-channeling of a high-power ultra short laser in matter, the propagation of a high-irradiance laser in a plasma creates an optional index depending nonlinearity on the light intensity and leads to new interesting nonlinear wave equation (see [3] [4] [5]). For more physical motivations and more references dealing with applications, we can refer to [6] [7] [8] [9] and references therein.

If we take $g^{2}(u)=1+\frac{\left(l^{\prime}\left(u^{2}\right)\right)^{2}}{2}$, Equation (1) can derive the corresponding equations with nonlocal term. For instance, If we set $g^{2}(u)=1+2 u^{2}$, i.e., $l(s)=s$, we get the superfluid film equation with nonlocal term in plasma physics:

$$
-\left(a+b \int_{\mathbb{R}^{N}}\left(1+2 u^{2}\right)|\nabla u|^{2} \mathrm{~d} x\right)\left[\Delta u+\Delta\left(u^{2}\right) u\right]+V(x) u=h(u), x \in \mathbb{R}^{N} .
$$


If we set $g^{2}(u)=1+\frac{u^{2}}{2\left(1+u^{2}\right)}$, i.e., $l(s)=(1+s)^{\frac{1}{2}}$, we get the equation:

$$
\begin{aligned}
& -\left(a+b \int_{\mathbb{R}^{N}}\left(1+\frac{u^{2}}{2\left(1+u^{2}\right)}\right)|\nabla u|^{2} \mathrm{~d} x\right)\left[\Delta u+\Delta\left(1+u^{2}\right)^{\frac{1}{2}} \frac{u}{2\left(1+u^{2}\right)^{\frac{1}{2}}} u\right]+V(x) u \\
& =h(u), x \in \mathbb{R}^{N},
\end{aligned}
$$

which is related to the model of the self-channeling of high-power ultrashort laser in matter.

Denote $H(u)=\int_{0}^{u} h(t) \mathrm{d} t$. We observe that the natural variational functional corresponding to Equation (1)

$$
I(u)=\frac{a}{2} \int_{\mathbb{R}^{N}} g^{2}(u)|\nabla u|^{2} \mathrm{~d} x+\frac{b}{4}\left(\int_{\mathbb{R}^{N}} g^{2}(u)|\nabla u|^{2} \mathrm{~d} x\right)^{2}+\frac{1}{2} \int_{\mathbb{R}^{N}} u^{2} \mathrm{~d} x-\int_{\mathbb{R}^{N}} H(u) \mathrm{d} x
$$

may be not well defined in $H^{1}\left(\mathbb{R}^{N}\right)$. Moreover, the set $\left\{u \in H^{1}\left(\mathbb{R}^{N}\right): \int_{\mathbb{R}^{N}} g^{2}(u)|\nabla u|^{2} \mathrm{~d} x<+\infty\right\}$ is not linear space. To overcome this difficulty, we make a change of variable constructed by Shen and Wang in [10], as $v=G(u):=\int_{0}^{u} g(t) \mathrm{d} t$.

Then we get

$$
\begin{aligned}
I(u)=J(v)= & \frac{a}{2} \int_{\mathbb{R}^{N}}|\nabla v|^{2} \mathrm{~d} x+\frac{b}{4}\left(\int_{\mathbb{R}^{N}}|\nabla v|^{2} \mathrm{~d} x\right)^{2} \\
& +\frac{1}{2} \int_{\mathbb{R}^{N}}\left|G^{-1}(v)\right|^{2} \mathrm{~d} x-\int_{\mathbb{R}^{N}} H\left(G^{-1}(v)\right) \mathrm{d} x .
\end{aligned}
$$

Under suitable assumptions on $g$ and $h$, we conclude that $J$ is well defined in $H^{1}\left(\mathbb{R}^{N}\right)$ and $J \in C^{1}$.

If $u$ is a nontrivial solution of (1), then it should satisfy

$$
\left(a+b \int_{\mathbb{R}^{N}}|\nabla u|^{2} \mathrm{~d} x\right) \int_{\mathbb{R}^{N}}\left[g^{2}(u) \nabla u \nabla \phi+g^{\prime}(u) g(u)|\nabla u|^{2} \phi+u \phi-h(u) \phi\right] \mathrm{d} x=0,
$$

for all $\phi \in C_{0}^{\infty}\left(\mathbb{R}^{N}\right)$. Let $\phi=\frac{1}{g(u)} \varphi,(2)$ is equivalent to

$$
\begin{aligned}
\left\langle J^{\prime}(v), \varphi\right\rangle= & \int_{\mathbb{R}^{N}}\left(a \nabla v \nabla \varphi+\frac{G^{-1}(v)}{g\left(G^{-1}(v)\right)} \varphi-\frac{h\left(G^{-1}(v)\right)}{g\left(G^{-1}(v)\right)} \varphi\right) \mathrm{d} x \\
& +b \int_{\mathbb{R}^{N}}|\nabla v|^{2} \mathrm{~d} x \int_{\mathbb{R}^{N}} \nabla v \nabla \varphi \mathrm{d} x \\
= & 0,
\end{aligned}
$$

for all $\varphi \in C_{0}^{\infty}\left(\mathbb{R}^{N}\right)$. Therefore, in order to find nodal solutions of (1), it suffices to study the following equation

$$
-\left(a+b \int_{\mathbb{R}^{N}}|\nabla v|^{2} \mathrm{~d} x\right) \Delta v+\frac{G^{-1}(v)}{g\left(G^{-1}(v)\right)}=\frac{h\left(G^{-1}(v)\right)}{g\left(G^{-1}(v)\right)}, \text { in } \mathbb{R}^{N} .
$$

Now, we consider the existence of nodal solutions of (3). Nonlocal problems like (3) have drawn a great deal of attention in recent years (see [11] [12] [13] [14] [15]). To begin with, Equation (3) can be derived as a nonlocal model for 
the vibrating string. It is related to the stationary analogue of equation

$$
\rho \frac{\partial^{2} u}{\partial t^{2}}-\left(\frac{P_{0}}{h}+\frac{E}{2 L} \int_{0}^{L}\left|\frac{\partial u}{\partial x}\right| \mathrm{d} x\right) \frac{\partial^{2} u}{\partial x^{2}}=f(x, u)
$$

proposed by Kirchhoff in [16] as an extension of the classical D'Alembert's wave equation for free vibrations of elastic strings. Kirchhoff's model takes into account the changes in length of the string produced by transverse vibrations. Here, $\rho$ is the mass density, $\frac{P_{0}}{h}$ is the initial tension, $E$ is related to the intrinsic properties of the string, such as the Young's modulus of material and $L$ is the length of the string. In [17], it was pointed out that such problems as (3) may be applied to describe the growth and movement of a particular species.

Different from the above mentioned literatures, for example [12] [13] [15], we provide a new viewpoint motivated by [18] for solving the generalized quasilinear Schödinger-Kirchhoff type problem. In this paper, some suitable algebraic techniques are used to find solutions. Precisely, we derive nodal solutions of (1) by transforming it into (3) and establish Equation (2) is equivalent to a system. Then, we occur the existence of nodal solutions of this system. We not only prove multiplicity result for $N=3$, but also give information about the case that $N \geq 4$. We achieve our purpose by solving the following system respect to $(v, \mu) \in \mathbb{R}^{N} \times \mathbb{R}^{+}$:

$$
\left\{\begin{array}{l}
-\Delta v+\frac{G^{-1}(v)}{g\left(G^{-1}(v)\right)}=\frac{h\left(G^{-1}(v)\right)}{g\left(G^{-1}(v)\right)}, \\
\mu-a-b \mu^{\frac{N-2}{2}} \int_{\mathbb{R}^{N}}|\nabla v|^{2} \mathrm{~d} x=0 .
\end{array}\right.
$$

Recall that a node of a radial solution of (1) is a radius $\rho>0$ such that $u(x)=0$ with $|x|=\rho$. The main purpose in this paper is to prove the equivalent of (3) and (4).

Proposition 1. Problem (3) has least one radial solution $v \in H^{1}\left(\mathbb{R}^{N}\right)$ if and only if system (4) has at least one solution $(w, \mu) \in H^{1}\left(\mathbb{R}^{N}\right) \times \mathbb{R}^{+}$such that $w$ is radial. Moreover, $w$ and $v$ have the same number of nodes.

Proposition 2. Problem (1) has least one radial solution $u \in H^{1}\left(\mathbb{R}^{N}\right)$ if and only if problem (3) has at least one solution $v \in H^{1}\left(\mathbb{R}^{N}\right)$ such that $v$ is radial. Moreover, $v$ and $u$ have the same number of nodes.

In order to state our main result, we need the following hypotheses:

$\left(\mathrm{h}_{1}\right) h(t)=o(t)$ as $t \rightarrow 0$.

$\left(\mathrm{h}_{2}\right)$ There exists $C>0$ and $2<p<2^{*}=\frac{2 N}{N-2}$ such that $|h(t)| \leq C\left(1+g(t)|G(t)|^{p-1}\right)$ for all $t \in \mathbb{R}$.

$\left(\mathrm{h}_{3}\right)$ There exists $\delta>0$ such that for any $t>0$, there holds $(1+\delta) h(t) \leq G(t)\left(\frac{h(t)}{g(t)}\right)^{\prime}$, where $G(t)=\int_{0}^{t} g(s) \mathrm{d} s$. 
$\left(\mathrm{h}_{4}\right) h \in C^{1}(\mathbb{R})$ is an odd function, $h(t)>0$ for $t>0, g \in C^{1}(\mathbb{R})$ is an even positive function and $g^{\prime}(t) \geq 0$ for all $t \geq 0, g^{\prime}(t) t<g(t)$ for all $t \in \mathbb{R}$.

Under the assumptions $\left(\mathrm{h}_{1}\right)-\left(\mathrm{h}_{2}\right), g$ and $h$ possess many important properties. Readers can find them in [19].

Applying Proposition 1 and 2, we can prove the following theorems.

Theorem 3. Suppose that $\left(\mathrm{h}_{1}\right)-\left(\mathrm{h}_{4}\right)$ are satisfied. Then for any integer $k>0$ the following holds.

i) If $N=3$, (3) has a pair $v_{k}^{+}$and $v_{k}^{-}$of radial solutions for any $a, b>0$.

ii) If $N=4$, there exists $b_{0} \geq b_{k}>0$ with $b_{k \rightarrow 0_{+}}$such that (3) has a pair $v_{k}^{+}$ and $v_{k}^{-}$of radial solutions for any $a>0$ and $0<b<b_{k}$, has no nontrivial solution for any $a>0$ and $b \geq b_{0}$.

iii) If $N \geq 5$, there exists $\alpha_{0} \geq \alpha_{k}>0$, with $\alpha_{k} \rightarrow 0^{+}$such that (3) has two pair $v_{k, 1}^{ \pm}$and $v_{k, 2}^{ \pm}$of radial solutions if $a b^{\frac{2}{N-4}}<\alpha_{k}$, has a radial solution $v_{k}$ if $a b^{\frac{2}{N-4}}=\alpha_{k}$, and has no nontrivial solution if $a b^{\frac{2}{N-4}}>\alpha_{0}$.

Moreover, each solution $v_{k}$ obtained in i)-iii) has exactly $k$ nodes $0<\rho_{v_{k}}^{1}<\rho_{v_{k}}^{2}<\cdots<\rho_{v_{k}}^{k}<\infty$.

Theorem 4. Suppose that $\left(\mathrm{h}_{1}\right)-\left(\mathrm{h}_{4}\right)$ are satisfied. Then for any integer $k>0$ the following holds.

i) If $N=3$, (1) has a pair $u_{k}^{+}$and $u_{k}^{-}$of radial solutions for any $a, b>0$.

ii) If $N=4$, there exists $b_{0} \geq b_{k}>0$ with $b_{k \rightarrow 0_{+}}$such that (1) has a pair $u_{k}^{+}$ and $u_{k}^{-}$of radial solutions for any $a>0$ and $0<b<b_{k}$, has no nontrivial solution for any $a>0$ and $b \geq b_{0}$.

iii) If $N \geq 5$, there exists $\alpha_{0} \geq \alpha_{k}>0$, with $\alpha_{k} \rightarrow 0^{+}$such that (1) has two pair $u_{k, 1}^{ \pm}$and $u_{k, 2}^{ \pm}$of radial solutions if $a b^{\frac{2}{N-4}}<\alpha_{k}$, has a radial solution $u_{k}$ if $a b^{\frac{2}{N-4}}=\alpha_{k}$, and has no nontrivial solution if $a b^{\frac{2}{N-4}}>\alpha_{0}$.

Moreover, each solution $u_{k}$ obtained in i)-iii) has exactly $k$ nodes $0<\rho_{u_{k}}^{1}<\rho_{u_{k}}^{2}<\cdots<\rho_{u_{k}}^{k}<\infty$.

\section{Existence and Nonexistence of Solutions of (4)}

In this section, we devote to solve system (4). Firstly, we will show an essential result which will be used to conclude Theorem 3 .

Proposition 5. Suppose that $\left(\mathrm{h}_{1}\right)-\left(\mathrm{h}_{4}\right)$ are satisfied. Then for any integer $k>0$ the following holds.

i) If $N=3,(4)$ has a pair $\left(v_{k}^{+}, \mu_{k}^{+}\right)$and $\left(v_{k}^{-}, \mu_{k}^{-}\right)$of radial solutions for any $a, b>0$.

ii) If $N=4$, there exists $b_{0} \geq b_{k}>0$ with $b_{k \rightarrow 0_{+}}$such that (4) has a pair $\left(v_{k}^{+}, \mu_{k}^{+}\right)$and $\left(v_{k}^{-}, \mu_{k}^{-}\right)$of radial solutions for any $a>0$ and $0<b<b_{k}$, has no nontrivial solution for any $a>0$ and $b \geq b_{0}$.

iii) If $N \geq 5$, there exists $\alpha_{0} \geq \alpha_{k}>0$, with $\alpha_{k} \rightarrow 0^{+}$such that (4) has two 
pair $\left(v_{k}^{ \pm}, \mu_{k, 1}^{ \pm}\right)$and $\left(v_{k}^{ \pm}, \mu_{k, 2}^{ \pm}\right)$of radial solutions if $a b^{\frac{2}{N-4}}<\alpha_{k}$, has a radial solution $\left(v_{k}, \mu_{k}\right)$ if $a b^{\frac{2}{N-4}}=\alpha_{k}$, and has no nontrivial solution if $a b^{\frac{2}{N-4}}>\alpha_{0}$.

Moreover, the first component $v_{k}$ of every solution obtained in i)-iii) is radially symmetric and has exactly $k$ nodes $0<\rho_{v_{k}}^{1}<\rho_{v_{k}}^{2}<\cdots<\rho_{v_{k}}^{k}<\infty$.

Clearly, $(0, a)$ is a trivial solution of (4). Here a solution $(v, \mu) \in H^{1}\left(\mathbb{R}^{N}\right) \times \mathbb{R}^{+}$of $(4)$ is called a nontrivial one if $(v, \mu) \neq(0, a)$.

In order to obtain conclusion of Proposition 5, we first establish the result about existence of nodal solutions for the following nonlinear elliptic equation:

$$
-\Delta v+\frac{G^{-1}(v)}{g\left(G^{-1}(v)\right)}=\frac{h\left(G^{-1}(v)\right)}{g\left(G^{-1}(v)\right)}, \text { in } \mathbb{R}^{N} .
$$

Lemma 1. Suppose that $\left(\mathrm{h}_{1}\right)-\left(\mathrm{h}_{4}\right)$ are satisfied. Then for any integer $k>0$, there exists a pair $v_{k}^{+}$and $v_{k}^{-}$of radial solutions of $(5)$ with $v_{k}^{-}(0)<0<v_{k}^{+}(0)$, having exactly $k$ nodes $0<r_{1}^{ \pm}<r_{2}^{ \pm}<\cdots<r_{k}^{ \pm}<\infty$.

Proof. This theorem was proved in [19], here we omit the detail.

Let $\mathcal{A}$ be the set of solutions of (5) and define

$$
\beta=\inf \{S(v): v \in \mathcal{A} \backslash\{0\}\},
$$

where

$$
S(v)=\int_{\mathbb{R}^{N}}|\nabla v|^{2} \mathrm{~d} x, \forall v \in H^{1}\left(\mathbb{R}^{N}\right) .
$$

According to Theorem 2.1, we obtain that $\mathcal{A} \backslash\{0\} \neq \varnothing$ and $\beta$ is well defined.

Lemma 2. Suppose that $\left(\mathrm{h}_{1}\right),\left(\mathrm{h}_{2}\right)$ and $\left(\mathrm{h}_{4}\right)$ are satisfied. Then it holds $\beta>0$.

Proof. For any $v \in \mathcal{A} \backslash\{0\}$, one has the Pohozaev identity

$$
\frac{N-2}{2 N} \int_{\mathbb{R}^{N}}|\nabla v|^{2} \mathrm{~d} x+\frac{1}{2} \int_{\mathbb{R}^{N}}\left|G^{-1}(v)\right|^{2} \mathrm{~d} x=\int_{\mathbb{R}^{N}} H\left(G^{-1}(v)\right) \mathrm{d} x .
$$

From assumptions $\left(\mathrm{h}_{1}\right),\left(\mathrm{h}_{2}\right)$ and $\left(\mathrm{h}_{4}\right)$, we obtain $\left|G^{-1}(s) g\left(G^{-1}(s)\right)\right| \geq|s|$,

$$
\lim _{|s| \rightarrow 0} \frac{h\left(G^{-1}(s)\right)}{G^{-1}(s)}=0 \text { and } \lim _{|s| \rightarrow \infty} \frac{h\left(G^{-1}(s)\right)}{g\left(G^{-1}(s)\right)|s|^{2^{*}-1}}=0 .
$$

For any $\epsilon>0$, there exists $C_{\epsilon}>0$ such that

$$
\left|\frac{h\left(G^{-1}(s)\right)}{g\left(G^{-1}(s)\right)}\right| \leq \epsilon\left|\frac{G^{-1}(s)}{g\left(G^{-1}(s)\right)}\right|+C_{\epsilon}|s|^{2^{*}-1}, \forall s \in \mathbb{R} .
$$

Obviously, this implies that $S(v) \leq C \int_{\mathbb{R}^{N}}|v|^{2^{*}} \mathrm{~d} x$ for some $C>0$. Applying Sobolev embedding theorem, we conclude $S(v) \leq C[S(v)]^{\frac{N}{N-2}}$, which establish that there exists a constant $\gamma>0$ such that $S(v) \geq \gamma$. Thus $\beta \geq \gamma>0$.

Proof of Proposition 5. This result was proved in ([18], Proposition 2.1). However, it plays a key role in this paper and for the sake of completeness and convenience ro reader, we here give the detail. For any $v \in H^{1}\left(\mathbb{R}^{N}\right) \backslash\{0\}$, define a 
function in $\mathbb{R}^{+}$given by

$$
f_{v}(\mu)=\mu-a-b \mu^{\frac{N-2}{2}} S(v), \forall \mu \in \mathbb{R}^{+} .
$$

Case 1: $N=3$. Theorem 5 implies that for any integer $k>0$, problem (5) has a pair $v_{k}^{+}$and $v_{k}^{-}$of radial solutions with exactly $k$ nodes $0<r_{1}^{ \pm}<r_{2}^{ \pm}<\cdots<r_{k}^{ \pm}<\infty$. Since $N=3$, we conclude

$$
f_{v_{k}^{ \pm}}(\mu)=\mu-a-b \mu^{\frac{1}{2}} S\left(v_{k}^{ \pm}\right),
$$

which implies that $\lim _{\mu \rightarrow+\infty} f_{v_{k}^{ \pm}}(\mu)=+\infty$ for any $a, b>0$. Noting that $f_{v_{k}^{ \pm}}(\mu)<0$ for all $\mu \in(0, a]$, there exists $\mu_{k}^{ \pm}>a$ such that $f_{v_{k}^{ \pm}}\left(\mu_{k}^{ \pm}\right)=0$. Thus $\left(v_{k}^{ \pm}, \mu_{k}^{ \pm}\right)$is a pair of solutions of (3) and conclusion i) holds.

Case 2: $N=4$. In the case, we have

$$
f_{v}(\mu)=(1-b S(v)) \mu-a,
$$

for all $(v, \mu) \in H^{1}\left(\mathbb{R}^{4}\right) \times \mathbb{R}^{+}$. For any integer $k>0$, let $v_{k}^{ \pm}$be a pair of radial solutions of (5) with exactly $k$ nodes $0<r_{1}^{ \pm}<r_{2}^{ \pm}<\cdots<r_{k}^{ \pm}<\infty$, $b_{k}=\min \left\{\frac{1}{S\left(v_{k}^{+}\right)}, \frac{1}{S\left(v_{k}^{-}\right)}\right\}$, and $b_{0}=\frac{1}{\beta}$, where $\beta$ is given in Lemma 2. Then $b_{k} \leq b_{0}$. Let $B_{i}=\left\{x \in \mathbb{R}^{4}: r_{i-1}<|x|<r_{i}\right\}$ and $v_{k, i}^{ \pm}=v_{k}^{ \pm}$in $B_{i}, v_{k, i}^{ \pm}=0$ on $\mathbb{R}^{N} \backslash B_{i}, 1=1,2, \cdots, k$. Then $v_{k, i}^{ \pm}, i=1,2, \cdots, k$, are nontrivial solutions of (5), which implies that $S\left(v_{k}^{ \pm}\right)=\sum_{i=1}^{k} S\left(v_{k, i}^{ \pm}\right) \geq k \beta$ and hence $b_{k} \rightarrow 0^{+}$as $k \rightarrow \infty$. For any $a>0$ and $0<b<b_{k}$, taking $\mu_{k}^{ \pm}=\frac{a}{1-b S\left(v_{k}^{ \pm}\right)}$, then one has $\mu_{k}^{ \pm}>a$ and $f_{v_{k}^{ \pm}}\left(\mu_{k}^{ \pm}\right)=0$. Thus $\left(v_{k}^{ \pm}, \mu_{k}^{ \pm}\right)$solve (2). However, for any $a>0$ and $b \geq b_{0}$, we have

$$
\sup _{v \in \mathcal{A} \backslash\{0\}} f_{v}(\mu) \leq\left(1-b_{0} \beta\right) \mu-a=-a<0,
$$

for all $\mu \in \mathbb{R}^{+}$, where $\mathcal{A}$ is defined in (6). This establishes that (4) has no nontrivial solution if $a>0$ and $b \geq b_{0}$.

Case 3: $N=5$. For each $v \neq 0$, we see that $\frac{\mathrm{d}}{\mathrm{d} \mu} f_{v}(\mu)=1-\frac{N-2}{2} \mu^{\frac{N-4}{2}} S(v)$ and hence $f_{v}(\mu)$ has a unique maximum point

$$
\mu_{v}=\left(\frac{2}{(N-2) b S(v)}\right)^{\frac{2}{N-4}}>0 .
$$

Let $v_{k}^{ \pm}$be a pair of radial solution of (5) with exactly $k$ nodes. It is easy to check that $f_{v_{k}^{ \pm}}(\mu)<0$ for $\mu \in(0, a], \lim _{\mu \rightarrow+\infty} f_{v_{k}^{ \pm}}(\mu)=-\infty$ and

$$
\max _{\mu \in \mathbb{R}^{+}} f_{v_{k}^{ \pm}}(\mu)=f_{v_{k}^{ \pm}}\left(\mu_{v_{k}^{ \pm}}\right)=\left(\frac{N-4}{N-2}\right)\left(\frac{2}{(N-2) b S\left(v_{k}^{ \pm}\right)}\right)^{\frac{2}{N-4}}-a .
$$

Define $\alpha_{k}=\min \left\{\alpha_{k}^{+}, \alpha_{k}^{-}\right\}$, where 


$$
\alpha_{k}^{+}=\left(\frac{N-4}{N-2}\right)\left(\frac{2}{(N-2) S\left(v_{k}^{ \pm}\right)}\right)^{\frac{2}{N-4}} .
$$

From Case 2, we have $\alpha_{k} \rightarrow 0^{+}$as $k \rightarrow \infty$. Clearly, it holds $f_{v_{k}^{ \pm}}\left(\mu_{v_{k}^{ \pm}}\right)>0$ if $a b^{\frac{2}{N-4}}<\alpha_{k}$, which implies that there exists $\mu_{k, 1}^{ \pm} \in\left(a, \mu_{v_{k}^{ \pm}}\right)$and $\mu_{k, 2}^{ \pm} \in\left(\mu_{v_{k}^{ \pm}},+\infty\right)$ such that $f_{v_{k}^{ \pm}}\left(\mu_{k, 1}^{ \pm}\right)=0$ and $f_{v_{k}^{ \pm}}\left(\mu_{k, 2}^{ \pm}\right)=0$ if $a b^{\frac{2}{N-4}}<\alpha_{k}$.

Moreover, it is easy to verify that either $f_{v_{k}^{+}}\left(\mu_{v_{k}^{+}}\right)=0$ or $f_{v_{\bar{k}}^{-}}\left(\mu_{v_{k}^{-}}\right)=0$ if $a b^{\frac{2}{N-4}}=\alpha_{k}$.

Set

$$
\alpha_{0}=\left(\frac{N-4}{N-2}\right)\left(\frac{2}{(N-2) \beta}\right)^{\frac{2}{N-4}}
$$

where $\beta$ is given in (6). For any $a, b>0$ with $a b^{\frac{2}{N-4}}>\alpha_{0}$, we conclude that

$$
\sup _{v \in \mathcal{A}\{\{0\}} f_{v}(\mu) \leq \sup _{v \in \mathcal{A}\{\{0\}} f_{v}\left(\mu_{v}\right) \leq\left(\frac{N-4}{N-2}\right)\left(\frac{2}{(N-2) b \beta}\right)^{\frac{2}{N-4}}-a=\alpha_{0} b^{\frac{-2}{N-4}}-a<0 .
$$

for any $\mu \in \mathbb{R}^{+}$. This implies that (4) has no nontrivial solutions if $a b^{\frac{2}{N-4}}>\alpha_{0}$ and iii) holds.

\section{Proofs of Proposition 1 and 2}

Proof of Proposition 1. If (4) has a solution $(v, \mu) \in H^{1}\left(\mathbb{R}^{N}\right) \times \mathbb{R}^{+}$, then one has

$$
-\Delta v+\frac{G^{-1}(v)}{g\left(G^{-1}(v)\right)}=\frac{h\left(G^{-1}(v)\right)}{g\left(G^{-1}(v)\right)}, \text { in } \mathbb{R}^{N},
$$

at least in a weak sense, and

$$
\mu=a+b \mu^{\frac{N-2}{2}} \int_{\mathbb{R}^{N}}|\nabla v|^{2} \mathrm{~d} x
$$

Make a change of variable, as $w(x)=v\left(\mu^{-\frac{1}{2}} x\right)=v(y)$. Then, we conclude that

$$
\begin{aligned}
& -\left(a+b \int_{\mathbb{R}^{N}}|\nabla w|^{2} \mathrm{~d} x\right) \Delta w(x)+\frac{G^{-1}(w(x))}{g\left(G^{-1}(w(x))\right)} \\
& =-\mu^{-1}\left(a+b \mu^{\frac{N-2}{2}} \int_{\mathbb{R}^{N}}|\nabla v|^{2} \mathrm{~d} x\right) \Delta v(y)+\frac{G^{-1}(v(y))}{g\left(G^{-1}(v(y))\right)} \\
& =\frac{h\left(G^{-1}(v(y))\right)}{g\left(G^{-1}(v(y))\right)}=\frac{h\left(G^{-1}(w(x))\right)}{g\left(G^{-1}(w(x))\right)},
\end{aligned}
$$

which implies that $w$ is a solution of (3).

If (3) has a solution $w \in H^{1}\left(\mathbb{R}^{N}\right)$, then one has 


$$
-\left(a+b \int_{\mathbb{R}^{N}}|\nabla w|^{2} \mathrm{~d} x\right) \Delta w+\frac{G^{-1}(w)}{g\left(G^{-1}(w)\right)}=\frac{h\left(G^{-1}(w)\right)}{g\left(G^{-1}(w)\right)}, \text { in } \mathbb{R}^{N},
$$

at least in a weak sense.

Letting $\mu=a+b \int_{\mathbb{R}^{N}}|\nabla w|^{2} \mathrm{~d} x$ and $v(x)=w\left(\mu^{\frac{1}{2}} x\right)=w(y)$, then we have

$$
\mu=a+b \mu^{\frac{N-2}{2}} \int_{\mathbb{R}^{N}}|\nabla v| \mathrm{d} x
$$

and

$$
\begin{aligned}
& -\Delta v(x)+\frac{G^{-1}(v(x))}{g\left(G^{-1}(v(x))\right)}=-\mu \Delta w(y)+\frac{G^{-1}(w(y))}{g\left(G^{-1}(w(y))\right)} \\
& =-\left(a+b \int_{\mathbb{R}^{N}}|\nabla w|^{2} \mathrm{~d} x\right) \Delta w(y)+\frac{G^{-1}(w(y))}{g\left(G^{-1}(w(y))\right)} \\
& =\frac{h\left(G^{-1}(w(y))\right)}{g\left(G^{-1}(w(y))\right)}=\frac{h\left(G^{-1}(v(x))\right)}{g\left(G^{-1}(v(x))\right)},
\end{aligned}
$$

which implies that $(v, \mu) \in H^{1}\left(\mathbb{R}^{N}\right) \times \mathbb{R}^{+}$is a solution of (4). Moreover, it is evident that $v$ and $w$ have the same radial symmetry and sign.

Proof of Proposition 2. If (3) has a solution $v \in H^{1}\left(\mathbb{R}^{N}\right)$, then one has

$$
-\left(a+b \int_{\mathbb{R}^{N}}|\nabla v|^{2} \mathrm{~d} x\right) \Delta v+\frac{G^{-1}(v)}{g\left(G^{-1}(v)\right)}=\frac{h\left(G^{-1}(v)\right)}{g\left(G^{-1}(v)\right)}, \text { in } \mathbb{R}^{N} .
$$

at least in a weak sense. Letting $u=G^{-1}(v)$ and combining the assumptions on $g$ and $h$, then we conclude that

$$
\left(a+b \int_{\mathbb{R}^{N}} g^{2}(u)|\nabla u|^{2} \mathrm{~d} x\right)\left[-\operatorname{div}\left(g^{2}(u) \nabla u\right)+g^{\prime}(u) g(u)|\nabla u|^{2}\right]+u=h(u) \text {, in } \mathbb{R}^{N} .
$$

which implies that $u \in H^{1}\left(\mathbb{R}^{N}\right)$ is a solution of (1). Moreover, by the definition of $G(t)$, it is obvious that $u$ and $v$ have the same radial symmetry and sign.

\section{Conclusion}

By the proof of Proposition 1 and 2, we establish problem (1) has least one radial solution $v \in H^{1}\left(\mathbb{R}^{N}\right)$ if and only if system (4) has at least one solution $(w, \mu) \in H^{1}\left(\mathbb{R}^{N}\right) \times \mathbb{R}^{+}$such that $w$ is radial. And $w$ and $v$ have the same number of nodes. Proving the existence of nodal solutions of problem (1) is equivalent to solve problem (4). In Section 2, we show the existence and nonexistence of solutions of (4). Combining the above statements the conclusions of Theorem 3 follow directly from Proposition 1 and 5. Similarly, the conclusions of Theorem 4 follow directly from Proposition 1, 2 and 5.

\section{Conflicts of Interest}

The author declares no conflicts of interest regarding the publication of this paper. 


\section{References}

[1] Cuccagna, S. (2009) On Instability of Excited States of Boblinear Schrödinger Equation. Physica D: Nonlinear Phenomena, 238, 38-54. https://doi.org/10.1016/j.physd.2008.08.010

[2] Kurihara, S. (1981) Large-Ampliyude Quasi-Solitons in Superfluid Films. Journal of the Physical Society of Japan, 50, 3262-3267. https://doi.org/10.1143/JPSJ.50.3262

[3] Brandi, H., Manus, C., Mainfray, G., Lehner, T. and Bannuad, G. (1993) Relativistic and Pondermotive Self-Focusing of a Laser Beam in a Radially Inhomogeneous Plasma. Physics of Fluids B: Plasma Physics, 5, 3539-3550.

https://doi.org/10.1063/1.860828

[4] De Bouard, A., Hayashi, N. and Saut, J. (1997) Global Existence of Small Solutions to a Relativistic Nonlinear Schrödinger Equation. Communications in Mathematical Physics, 189, 73-105. https://doi.org/10.1007/s002200050191

[5] Ritchie, B. (1994) Relativistic Self-Focusing and Channel Formation in Laser-Plasma Interactions. Physical Review E, 50, R687(R). https://doi.org/10.1103/PhysRevE.50.R687

[6] Bass, F.G. and Nasanov, N.N. (1990) Nonlinear Electromagnetic-Spin Waves. Physics Reports, 189, 165-223. https://doi.org/10.1016/0370-1573(90)90093-H

[7] Brüll, L. and Lange, H. (1986) Solitary Waves for Quasilinear Schrödinger Equations. Expositiones Mathematicae, 4, 279-288. https://doi.org/10.1080/00036818608839619

[8] Poppenberg, M., Schmitt, K. and Wang, Z. (2002) On the Existence of Soliton Solutions to Quasilinaer Schrödinger Equations. Calculus of Variations and Partial Differential Equations, 14, 329-344. https://doi.org/10.1007/s005260100105

[9] Lange, H., Poppenberg, M. and Teismann, H. (1999) Nash-Moser Methods for the Solution of Quasilinaer Schrödinger Equations. Communications in Partial Differential Equations, 24, 1399-1418. https://doi.org/10.1080/03605309908821469

[10] Shen, Y. and Wang, Y. (2013) Soliton Solutions for a Generalized Quasilinear Schrödinger Equations. Nonlinear Analysis, Theory, Methods and Applications, 80, 194-201. https://doi.org/10.1016/j.na.2012.10.005

[11] Chen, C.Y., Kuo, Y.C. and Wu, T.F. (2011) The Nehari Manifold for a Kirchhoff Type Problem Involving Sign-Changing Weight Functions. Journal of Differential Equations, 250, 1876-1908. https://doi.org/10.1016/j.jde.2010.11.017

[12] He, X. and Zou, W. (2016) Existence and Concentration Result for the Fractional Schrödinger Equations with Critical Nonlinearities. Calculus of Variations and Partial Differential Equations, 55, 1-39. https://doi.org/10.1007/s00526-016-1045-0

[13] He, Y., Li, G. and Peng, S. (2014) Concentrating Bound States for Kirchhoff Type Problems in $\mathrm{R}^{3}$ Involving Critical Sobolev Exponents. Advanced Nonlinear Studies, 14, 483-510. https://doi.org/10.1515/ans-2014-0214

[14] Li, G. and Ye, H. (2014) Existence of Positive Ground State Solutions for the Nonlinear Kirchhoff Type Equations in $\mathrm{R}^{3}$. Journal of Differential Equations, 257, 566-600. https://doi.org/10.1016/j.jde.2014.04.011

[15] Tang, X.H. and Cheng, B. (2016) Ground State Sign-Changing Solutions for Kirchhoff Type Problems in Bounded Domains. Journal of Differential Equations, 261, 2384-2402. https://doi.org/10.1016/j.jde.2016.04.032

[16] Kirchhoff, G. (1883) Vorlesungen Über Mechanik Birkhäuser Basel.

[17] Pucci, P., Xiang, M. and Zhang, B. (2015) Multiple Solutions for Nonhomogeneous Schrödinger-Kirchhoff Type Equations Involving the Fractional $p$-Laplacian in $\mathrm{R}^{\mathrm{N}}$. Calculus of Variations and Partial Differential Equations, 54, 2785-2806. 
https://doi.org/10.1007/s00526-015-0883-5

[18] $\mathrm{Wu}, \mathrm{K}$. and Zhou, F. (2019) Nodal Solutions for a Kirchhoff Type Problem in $\mathrm{R}^{\mathrm{N}}$. Applied Mathematics Letters, 55, 58-63. https://doi.org/10.1016/j.aml.2018.08.008

[19] Deng, Y., Peng, S. and Wang, J. (2014) Nodal Soliton Solutions for Generalized Quasilinear Schrödinger Equations. Journal of Mathematical Physics, 55, 349-381. https://doi.org/10.1063/1.4874108 